\title{
Response of Superconductor Bolometer to Phonon Fluxes
}

\author{
B.A. Danilchenko ${ }^{a}, \mathrm{Cz}$. Jasiukiewicz ${ }^{b}$, T. PAszkiewicz $^{c}$ \\ AND S. WOLSKI ${ }^{c}$ \\ ${ }^{a}$ Institute of Physics, National Academy of Sciences of Ukraine \\ Prospect Nauki 46, 252650 Kiev, Ukraine \\ ${ }^{b}$ Chair of Physics, Rzeszów University of Technology \\ Pola 2, 35-959 Rzeszów, Poland \\ ${ }^{c}$ Institute of Physics, University of Rzeszów \\ Rejtana 16A, 35-310 Rzeszów, Poland
}

(Received September 30, 2002; revised version December 9, 2002; in final form February 20, 2003)

A metallic film bolometer with heat capacity $C$ is in contact with thermal bath and with crystalline specimen and is biased by a constant current $I_{\mathrm{b}}$. The thermal contact of the bolometer is characterized by the thermal conductance $G$. The bolometer operates in the linear regime of dependence of resistance on temperature characterized by a constant $\alpha$. Experiments which allow one to measure $\alpha, C$, and $G$ are proposed. The characteristic time $\tau=C / G$ and characteristic current $I_{\mathrm{m}}=\sqrt{G / \alpha}$ affect the effective relaxation rate $A$ of the bolometer resistance $R_{\mathrm{b}}(t)$. The knowledge of the power $W(t)$ absorbed by detector allows one to calculate $R_{\mathrm{b}}(t)$. The inverse problem of calculation of $W(t)$ from known $R_{\mathrm{b}}(t)$ is also solved. The suitable algorithms are proposed. Deconvoluted absorbed power is obtained for experiments performed on GaAs and compared with phonoconductivity signal of two-dimensional electron gas structure as well as with results of Monte Carlo computer experiments.

PACS numbers: 07.57.Kp, 02.30.Hq, 66.70.+f

\section{Introduction}

Heat pulses become the standard tool used for studying properties of crystalline dielectrics, semiconductors, and superconductors. Pulse lengths used are short enough to allow to resolve different kinds of phonons and propagation directions in millimeter sized samples. Edwards and collaborators discussed the use of superconducting bolometers for detecting phonon pulses in the regime in 
which their lengths are shorter than the bolometer response time [1]. On the other hand, we studied the dependence of the form of phonon pulses on the direction of propagation and on the dimensions of the source as well as on the detector area [2]. The response of superconducting bolometer to electromagnetic radiation was considered by Fuson [3]. Some authors used metallic film bolometers in normal, non-superconducting state (cf. Ref. [4]). Our description is valid for both kinds of metallic thin film bolometers.

Usually linear changes of the signal voltage of thin-metallic film superconducting bolometers (TFSB) due to absorption of phonon pulses under condition of constancy of the biasing current are considered (cf. [1,3]). In particular in a series of papers, using the Laplace transform technique, Sherlock and Wyatt studied this linear regime in a very detailed way (references to their papers can be found in Ref. [5]). We also here restrict ourselves to the linear approximation.

Wyatt and collaborators (cf. [5]) were mainly interested in heat pulses propagating in superfluid helium. Here we describe experiments with the use of quasiparticles-phonons propagating in crystalline solids in the form of pulses, carrying energy and quasimomentum. A metallic film is the measuring device. The energy imparted to the specimen is known and controlled. An experimenter is interested in the time resolved bolometer signal. As a rule the total amount of energy falling onto detector is not measured (in the time-of-flight experiments, with which we deal, only ratios of pulses heights are important). In the described experiments the value of proportionality constant $\alpha$, relating changes of resistance and temperature, should be moderate. The detailed description of the time-of-flight and imaging techniques is given by Northrop and Wolfe [6] and Wolfe [7].

There exists a different class of experiments with the use of superconducting metallic films, namely experiments on the calorimetric detection of elementary particles and radiation ( $\mathrm{cf}$. [8] and [9]). In these experiments a superconducting edge sensor is an active element of the measuring device - a microcalorimeter. Such sensor operates in the condition of constant bias voltage. The electro-thermal feedback makes possible to keep temperature of the sensor constant. The output of microcalorimeter is the time integral of the absorbed energy. In experiments with microcalorimeters values of parameter $\alpha$ are rather large. One may say that in experiments describing by us the time resolution is optimized, whereas in experiments on detection of elementary particles the energy resolution should be optimal.

In this paper we derive basic linear differential equation for the resistance metallic film bolometers irradiated by phonon fluxes under assumption of constancy of the biasing current. The general solution of this equation is obtained. We also discuss its application for deconvolution of the output bolometer signal to give the incident phonon fluxes. The inverse problem - for a given time dependent energy flux to find the bolometer signal - is also solved. 


\section{Differential equation for bolometer resistance}

\subsection{General considerations}

Let us consider the bolometer and the specimen in contact with thermal bath of temperature $T_{\mathrm{s}}$. The thermal capacity of the bolometer at the ambient temperature is $C=C\left(T_{\mathrm{s}}\right)$. This thermal contact is characterized by the heat leak constant $G$. The source of phonons is placed in the origin of the Cartesian coordinate system. The $z$-axis of this coordinate system is directed towards the detector. Thus, only phonons with positive $z$-components of velocities reach the bolometer. The radius vector $r$ of the center of detector has the length $r$ and the direction $\hat{r}$ defined by two angles $\left(\theta_{r}, \phi_{r}\right)$. The surface area of the detector is $\Delta S_{\mathrm{d}}$, while $\hat{\boldsymbol{n}}_{\mathrm{d}}$ is the vector normal to it. When viewed from the source the detector subtends the body angle $\Delta \Omega_{\mathrm{d}}=\Delta S_{\mathrm{d}}\left[\hat{n}_{\mathrm{d}} \hat{r}\right] / r^{2}$. The absorption of phonon flux of the power $W_{\pi}(t)$ falling upon the bolometer receiving surface causes the change in the temperature of the bolometer and, consequently, the change $\Delta R_{\mathrm{b}}$ in its resistance $R_{\mathrm{b}}$. Let the assumptions be made that the bolometer receiving surface is small enough for the energy flux to fall uniformly, that there are no appreciable temperature gradients in the bolometer, no radiation losses, no conduction losses through the leadwires. The instantaneous temperature $T(t)$ of the bolometer obeys an ordinary nonautonomous differential equation, which generally is nonlinear in $T(t)$. In the case when the polarizing current is controlled and in the linear approximation this equation has the form (cf. $[1,3])$ :

$$
C \frac{\mathrm{d}(t)}{\mathrm{d} t}-W_{\mathrm{J}}(t)+G\left[T(t)-T_{\mathrm{s}}\right]=W_{\pi}(t) .
$$

The Joule heating term $W_{\mathrm{J}}(t)$ should be written in terms of variables related to experimental conditions. In the case of experiments in which the polarizing current $I_{\mathrm{b}}$ is controlled and the resistance $R_{\mathrm{b}}(t)$ is the measured quantity

$$
W_{\mathbf{J}}(t)=I_{\mathrm{b}}^{2} R_{\mathrm{b}}(t)
$$

In experiments in which the voltage $V$ is controlled and the measured quantity is the instantaneous current through the bolometer $I(t)[8,9]$ :

$$
\begin{aligned}
W_{\mathbf{J}}(t)= & I(t) V . \\
& \text { 2.2. Experiments in which the resistance is measured }
\end{aligned}
$$

In our recent experiments the biasing current is fixed and the measured quantity is the resistance of the bolometer [10]. A DC bias current is supplied by a coaxial cable. The same cable is also used for the transmission of bolometer signals. These signals are separated by T-junction and via a blocking capacitor $C$ they are passed to the broad-band amplifier. The circuit diagram for the superconducting 


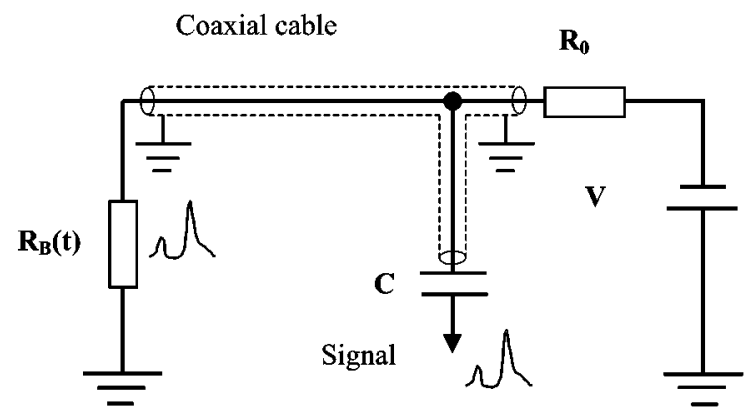

Fig. 1. The diagram of the electric circuit used in our experiments on the bolometer response.

bolometer used in the experiments presented here is depicted in Fig. 1. The same method was used in our previous experiments [11]. It allowed us to achieve the time response of the bolometer in the range of 25 ns.

The bias current is $I_{\mathrm{b}}=V /\left[R_{0}+R_{\mathrm{b}}\left(T_{\mathrm{s}}\right)\right]$. As a rule $R_{0} \gg R_{\mathrm{b}}\left(T_{\mathrm{s}}\right)$. Let us suppose that the bolometer absorbed the phonon flux and, in consequence, changed its resistance from $R_{\mathrm{b}}\left(T_{\mathrm{s}}\right)$ to $R_{\mathrm{b}}(t)=R_{\mathrm{b}}\left(T_{\mathrm{s}}\right)+\Delta R_{\mathrm{b}}[T(t)]$. Hence, also $I_{\mathrm{b}}$ has to change by $\Delta I_{\mathrm{b}}$ :

$$
\left|\Delta I_{\mathrm{b}}\right|=\left|I_{\mathrm{b}}-\frac{V}{R_{0}+R_{\mathrm{b}}\left(T_{\mathrm{s}}\right)+\Delta R_{\mathrm{b}}[T(t)]}\right| \cong I_{\mathrm{b}} \frac{\Delta R_{\mathrm{b}}}{R_{0}} .
$$

For superconducting bolometer and strong phonon fluxes $\Delta R_{\mathrm{b}} \approx 1 \div 10 \Omega$, whereas $R_{0} \approx 10^{4} \div 10^{5} \Omega$, and $R_{\mathrm{b}}\left(T_{\mathrm{s}}\right) \approx 50 \Omega$, so

$$
\frac{\left|\Delta I_{\mathrm{b}}\right|}{I_{\mathrm{b}}} \approx 10^{-5} \div 10^{-3}
$$

and

$$
\frac{\Delta R_{\mathrm{b}}}{R_{\mathrm{b}}\left(T_{\mathrm{s}}\right)} \approx 2 \times 10^{-2} \div 2 \times 10^{-1}
$$

This means that the second term of Eq. (1) can be approximated

$$
I^{2}(t) R_{\mathrm{b}}[T(t)] \cong I_{\mathrm{b}}^{2} R_{\mathrm{b}}\left(T_{\mathrm{s}}\right)\left\{1+\frac{\Delta R_{\mathrm{b}}[T(t)]}{R_{\mathrm{b}}}\right\}
$$

where $I(t)$ is the instantaneous current and $R_{\mathrm{b}}(t)$ is the instantaneous bolometer resistance.

The resistance $R_{\mathrm{b}}$ is the function of biasing current and temperature $R_{\mathrm{b}}=$ $R_{\mathrm{b}}\left(I_{\mathrm{b}}, T\right)$. Inverting this relation we can find $I[T(t), R(t)]$. Our estimates show that the current variations are small (cf. also Ref. [6]). Therefore one may assume that $I[T(t), R(t)]=$ const. Hence,

$$
\left(\frac{\partial I_{\mathrm{b}}}{\partial T}\right)_{R} \frac{\partial T}{\partial t}+\left(\frac{\partial I_{\mathrm{b}}}{\partial R}\right)_{T} \frac{\partial R}{\partial t}=0
$$


This allows to express the time derivative of temperature by time derivative of the resistance

$$
\frac{\partial T}{\partial t}=-\frac{\left(\frac{\partial I_{\mathrm{b}}}{\partial R}\right)_{T}}{\left(\frac{\partial I_{\mathrm{b}}}{\partial T}\right)_{R}} \frac{\partial R}{\partial t}
$$

Using identities valid for Jacobians the coefficient multiplying the time derivative of the resistance can be written in a simpler form

$$
-\frac{\left(\frac{\partial I_{\mathrm{b}}}{\partial R}\right)_{T}}{\left(\frac{\partial I_{\mathrm{b}}}{\partial T}\right)_{R}}=-\frac{\frac{\partial\left(I_{\mathrm{b}}, T\right)}{\partial(R, T)}}{\frac{\partial\left(I_{\mathrm{b}}, R\right)}{\partial(T, R)}}=\frac{\frac{\partial\left(I_{\mathrm{b}}, T\right)}{\partial(T, R)}}{\frac{\partial\left(I_{\mathrm{b}}, R\right)}{\partial(T, R)}}=\frac{\partial\left(I_{\mathrm{b}}, T\right)}{\partial\left(I_{\mathrm{b}}, R\right)}=\left(\frac{\partial T}{\partial R}\right)_{I_{\mathrm{b}}}=\left[\left(\frac{\partial R}{\partial T}\right)_{I_{\mathrm{b}}}\right]^{-1}
$$

The derivative of resistance is calculated for $T=T_{c}$. Now the first term of Eq. (1) takes the form

$$
\frac{\mathrm{d} T(t)}{\mathrm{d} t}=\frac{1}{\left(\frac{\partial R_{\mathrm{b}}}{\partial T}\right)_{I_{\mathrm{b}}}} \frac{\mathrm{d} R_{\mathrm{b}}(t)}{\mathrm{d} t}
$$

\subsubsection{Superconducting bolometers}

An example of $R-I$ characteristics for the granular aluminum superconducting bolometer and for various temperatures is plotted in Ref. [1]. As a rule the superconducting bolometer is stabilized at the transition where the resistance is most sensitive to temperature, which means that

$$
\left(\mathrm{d} R_{\mathrm{b}} / \mathrm{d} T\right)_{T=T_{\mathrm{c}}} \sim \alpha,
$$

where $\alpha$ is a constant - the bolometer sensitivity parameter. We shall denote this coefficient for superconductors by $\alpha_{\mathrm{s}}$. As a rule $\alpha_{\mathrm{s}} \cong 10^{2} \Omega / \mathrm{K}$, however $\alpha_{\mathrm{s}} \cong 10^{3} \Omega / \mathrm{K}$ was reported [7]. Let us introduce a characteristic time $[1,3] \tau$ and a characteristic current [3] $I_{\mathrm{m}}$ :

$$
\tau=\frac{C}{G}, \quad I_{\mathrm{m}}=\sqrt{\frac{G}{\alpha}} .
$$

Now we may transform Eq. (1) to the differential equation for the resistance $R_{\mathrm{b}}(t)$ :

$$
\frac{\mathrm{d} R_{\mathrm{b}}(t)}{\mathrm{d} t}-\Lambda^{-1} R_{\mathrm{b}}(t)=\frac{R_{\mathrm{b}}(t)}{\tau}+\frac{1}{I_{\mathrm{b}}^{2} \tau}\left(\frac{I_{\mathrm{b}}}{I_{\mathrm{m}}}\right)^{2} W_{\boldsymbol{r}}(t),
$$

where

$$
\Lambda=\tau /\left[1-\left(I_{\mathrm{b}} / I_{\mathrm{m}}\right)^{2}\right]
$$

is the characteristic relaxation time which depends on both the characteristic time $\tau$ and the bias current $I_{\mathrm{b}}[3]$. This time is positive when $I_{\mathrm{m}}>I_{\mathrm{b}}$. Thus, the bias current cannot exceed the critical maximum value $I_{\mathrm{m}}(5)$. The second term on right hand side (rhs) of Eq. (6) has the proper dimension. Namely, we can write it in the form 


$$
\left[\frac{W_{r}(t)}{I_{\mathrm{m}}^{2} \rho} \frac{\rho}{\tau}\right]=\left[\frac{\rho}{\tau}\right]
$$

where $\rho$ is an arbitrary quantity with the dimension of resistance. We shall underline that the time constant of the electric circuit is of the order of 5 ns, i.e. is much shorter than both $\tau$ and $\Lambda$.

\section{Determination of differential equation parameters}

Since the thin metallic films used as the bolometers are nonuniform and generally non-ideal, the basic parameters characterizing them, namely $\alpha, C_{\mathrm{V}}$, and $G$ cannot be calculated. Therefore, we briefly describe several experiments in which they can be measured. In fact, the differential equation for resistance $R_{\mathrm{b}}(t)$ (6) depends only on two quantities characterizing the response of the bolometer, namely on $I_{\mathrm{m}}$ (Eq. (5)) and on $\Lambda$ (Eq. (7)).

\subsection{Stationary state in the absence of the source of external power}

Let us consider a stationary state in the absence of phonon flux, so the bolometer is heated to a temperature $T>T_{\mathrm{s}}$ only by the bias current. From Eq. (6) we get

$$
R_{\mathrm{b}}^{(\text {stat })}=\frac{R_{\mathrm{b}}\left(T_{\mathrm{s}}\right)}{1-\left(\frac{I_{\mathrm{b}}}{I_{\mathrm{m}}}\right)^{2}} .
$$

The resistance $R_{\mathrm{b}}^{\text {(stat) }}$ should fall inside the range of constant $\mathrm{d} R(T) / \mathrm{d} T$, the range within which this development is valid. This means that the bias current should be small enough. Equation (8) can be written as

$$
R_{\mathrm{b}}^{(\text {stat })}=1 /\left[1-\left(I_{\mathrm{b}} / I_{\mathrm{m}}\right)^{2}\right] R_{\mathrm{b}}\left(T_{\mathrm{s}}\right)=\frac{\Lambda}{\tau} R_{\mathrm{b}}\left(T_{\mathrm{s}}\right) .
$$

If $I_{\mathrm{b}} / I_{\mathrm{m}} \ll 1$, then for small bias currents the change $\Delta R_{\mathrm{b}}^{\text {(stat) }} \equiv R_{\mathrm{b}}^{\text {(stat) }}-R_{\mathrm{b}}\left(T_{\mathrm{s}}\right)$ is proportional to the ratio $\left(I_{\mathrm{b}} / I_{\mathrm{m}}\right)^{2}$ :

$$
\Delta R_{\mathrm{b}}^{\text {(stat) }} \cong R_{\mathrm{b}}\left(T_{\mathrm{s}}\right)\left(\frac{I_{\mathrm{b}}}{I_{\mathrm{m}}}\right)^{2} .
$$

Using Eq. (8) with the help of the plot of the dependence of resistance against DC current for a given ambient temperature $T_{\mathrm{s}}$ and the given bias current $I_{\mathrm{b}}$, we may find the coefficient $I_{\mathrm{m}}=\sqrt{G / \alpha}$. Since the coefficient $\alpha$ can be determined from the linear part of the temperature-resistance characteristics of the thin metal film used as the bolometer, one can deduce the value of $G$. 


\subsection{General solution of the differential equation for resistance} form

Using the definition of $R_{\mathrm{b}}^{\text {(stat) }}$ (Eq. (8)) we may write Eq. (6) in a simple

$$
\frac{\mathrm{d} \delta R_{\mathrm{b}}(t)}{\mathrm{d} t}+\frac{1}{\Lambda} \delta R_{\mathrm{b}}(t)=\frac{1}{I_{\mathrm{b}}^{2} \tau}\left(\frac{I_{\mathrm{b}}}{I_{\mathrm{m}}}\right)^{2} W_{r}(t),
$$

where $\delta R_{\mathrm{b}}(t) \equiv R_{\mathrm{b}}(t)-R_{\mathrm{b}}^{(\text {stat })}$. Let us multiply both sides of Eq. (9) by $\exp (t / A)$, then it can be written in a useful form

$$
\frac{\mathrm{d}}{\mathrm{d} t}\left[\mathrm{e}^{t / \Lambda} \delta R_{\mathrm{b}}(t)\right]=\mathrm{e}^{t / \Lambda} \frac{1}{I_{\mathrm{b}}^{2} \tau}\left(\frac{I_{\mathrm{b}}}{I_{\mathrm{m}}}\right)^{2} W_{r}(t) .
$$

One can find the solution of this equation with the initial condition $\delta R_{\mathrm{b}}(t=0)=$ $\delta R_{\mathrm{b}}^{(0)}$ :

$$
\delta R_{\mathrm{b}}(t)=\mathrm{e}^{-t / \Lambda} \delta R_{\mathrm{b}}{ }^{(0)}+\bar{R}_{W}(r, t)
$$

where

$$
\bar{R}_{W}(r, t)=\frac{1}{I_{\mathrm{b}}^{2} \tau}\left(\frac{I_{\mathrm{b}}}{I_{\mathrm{m}}}\right)^{2} \int_{0}^{t} \mathrm{~d} t^{\prime} \mathrm{e}^{-\left(t-t^{\prime}\right) / \Lambda} W_{r}\left(t^{\prime}\right)
$$

\subsection{Pulsed radiant power}

Let us assume that the constant flux $W_{r}$ is instantaneously turned in at $t=0$, i.e. $W_{\boldsymbol{r}}^{(\text {inf }- \text { step })}(t)=W_{\boldsymbol{r}} \theta(t)$. Then, the solution of Eq. (9) reads

$$
R_{\mathrm{b}}^{(\mathrm{inf}-\mathrm{step})}(t)=R_{\mathrm{b}}^{(0)} \mathrm{e}^{-t / A}+\left(R_{\mathrm{b}}^{(\mathrm{stat})}+\frac{W_{\boldsymbol{r}}}{I_{\mathrm{m}}^{2}-I_{\mathrm{b}}^{2}}\right)\left(1-\mathrm{e}^{-t / \Lambda}\right),
$$

where $R_{\mathrm{b}}^{(0)}=R_{\mathrm{b}}(t=0)$. The system disturbed in this way relaxes to a new stationary state $\left[R_{\mathrm{b}}^{\text {(stat) }}+W_{r} /\left(I_{\mathrm{m}}^{2}-I_{\mathrm{b}}^{2}\right)\right]$ defined by $\lim _{t \rightarrow \infty} R_{\mathrm{b}}^{(\text {inf-step })}(t)$.

Next, let us suppose that the disturbance $W_{\mathbb{T}}(t)$ is abruptly turned on at $t_{1}$ and then it is abruptly turned off at $t_{2}\left(t_{2}>t_{1}\right)$, i.e.

$$
W_{r}^{(\text {fin-step })}=W_{\boldsymbol{r}}\left[\theta\left(t-t_{1}\right)-\theta\left(t-t_{2}\right)\right] .
$$

In this case the solution of differential equation for $R_{\mathrm{b}}(t)$ reads

$$
\begin{gathered}
R_{\mathrm{b}}^{(\text {fin-step })}(t)=R_{\mathrm{b}}^{(\mathrm{stat})}+\left[R_{\mathrm{b}}^{(0)}-R_{\mathrm{b}}^{(\mathrm{stat})}\right] \mathrm{e}^{-t / \Lambda} \\
+\frac{W_{\boldsymbol{r}}}{I_{\mathrm{m}}^{2}-I_{\mathrm{b}}^{2}}\left[\mathrm{e}^{-\left(t-t_{1}\right) / \Lambda}-\mathrm{e}^{-\left(t-t_{2}\right) / \Lambda}\right] .
\end{gathered}
$$

Let us notice that in both considered cases the resistance relaxes with characteristic time $\Lambda$, which for $I_{\mathrm{m}}>I_{\mathrm{b}}$ is longer than $\tau$. Only for weak bias currents $\left(I_{\mathrm{b}} \ll I_{\mathrm{m}}\right)$ the relation $A \cong \tau$ is fulfilled. With growing $I_{\mathrm{b}}$ the characteristic time $\Lambda$ enlarges. If we already determined $I_{\mathrm{m}}$ and $G$, as a result of observation of the decay of $R_{\mathrm{b}}^{(\mathrm{inf}-\mathrm{step})}$ and $R_{\mathrm{b}}^{(\mathrm{fin}-\mathrm{step})}$ we are able to determine the characteristic time $\Lambda$, and thus, also the characteristic time $\tau$. Knowing the constant $G$ in this way we can determine the heat capacity $C$ of the film used as the detector of phonons. 


\section{Algorithms of numerical calculations}

Let us consider $\delta R_{\mathrm{b}}(t+\delta t)$, according to Eqs. (10) and (11):

$$
\begin{array}{r}
\delta R_{\mathrm{b}}(t+\delta t)=\exp [-(t+\delta t) / \Lambda]\left[\delta R_{\mathrm{b}}^{(0)}+\frac{1}{\tau I_{\mathrm{m}}^{2}} \int_{0}^{t+\delta t} \mathrm{~d} t \exp \left(t^{\prime} / \Lambda\right) W_{\boldsymbol{r}}\left(t^{\prime}\right)\right] \\
=\delta R_{\mathrm{b}}(t) \mathrm{e}^{-\delta t / \Lambda}+\frac{1}{\tau I_{\mathrm{m}}^{2}} \exp [-(t+\delta t) / \Lambda] \int_{t}^{t+\delta t} \mathrm{~d} t^{\prime} \exp \left(t^{\prime} / \Lambda\right) W_{r}\left(t^{\prime}\right) .
\end{array}
$$

For small $\delta t$ we can approximate the integral term using the trapezoidal rule

$$
\delta R_{\mathrm{b}}(t+\delta t) \cong \delta R_{\mathrm{b}}(t) \mathrm{e}^{-\delta t / \Lambda}+\frac{\delta t}{2 \tau I_{\mathrm{m}}^{2}}\left[W_{\boldsymbol{r}}(t+\delta t)-\exp (-\delta t / \Lambda) W_{\boldsymbol{r}}(t)\right] .
$$

In Sec. 5 we described the method of calculating $W_{\boldsymbol{r}}(t)$. This quantity can be also obtained as a result of computer experiments (cf. Ref. [12]). Equation (14) allows us to establish the explicit dependence of $\delta R_{\mathrm{b}}$ on time. Equation (14) yields the recurrence formula for $W_{r}$ :

$$
W_{\pi}(t+\delta t) \cong \exp (-\delta t / \Lambda) W_{\pi}(t)+\frac{2 \tau I_{\mathrm{m}}^{2}}{\delta t}\left[\delta R_{\mathrm{b}}(t+\mathrm{d} t)-\delta R_{\mathrm{b}}(t) \exp (-\delta t / \Lambda)\right] .
$$

One may expect that the formula (15) can be used for the purpose of deconvolution of $W_{r}(t)$ (we shall call the deconvolution also the direct problem). Unfortunately, it is numerically unstable, so rather the differential Eq. (9) should be used for this purpose. Measurements provide us with values of $\delta R_{\mathrm{b}}$ for various instants of time. Hence, using Eq. (9) we can establish how $W_{\boldsymbol{r}}(t)$ depends on time.

The inverse problem also can be solved. Let us suppose that one knows the phonon flux $W_{r}(t)$, say as the result of computer experiments (cf. Ref. [12]), or calculate it numerically (cf. Sec. 6). Then, using Eq. (15) one can fix the time dependence of $\delta R_{\mathrm{b}}$ and compare it with experimental findings. We shall call this procedure the convolution. From Eq. (9) it is seen that to obtain the explicit time dependence of phonon flux $W_{\boldsymbol{r}}(t)$ one should calculate the time derivative $\mathrm{d} \delta R_{\mathrm{b}}(t) / \mathrm{d} t$ of quantities which are determined in real or computer experiments. Therefore, as a rule the $\delta R_{\mathrm{b}}(t)$ curve is quite noisy. Thus, the first term of Eq. (9) is even more noisy. The noise of $\delta R_{\mathrm{b}}(t)$ can be reduced in the way used in the computer graphics. Let us suppose that the set of numbers $\delta R_{\mathrm{b}}^{(i)}=\delta R_{\mathrm{b}}\left(t_{i}\right)$ $(i=1, \ldots, n)$ is given. Now let us define the rounded set $\delta \bar{R}_{\mathrm{b}}^{(i)}$ of $\delta R_{\mathrm{b}}\left(t_{i}\right)$ :

$$
\begin{aligned}
& \delta \bar{R}_{\mathrm{b}}^{(i)}=\frac{1}{4} \delta R_{\mathrm{b}}^{(i-1)}+\frac{1}{2} R_{\mathrm{b}}^{(i)}+\frac{1}{4} \delta R_{\mathrm{b}}^{(i+1)}, \quad i=2, \ldots, n-1, \\
& \delta R_{\mathrm{b}}^{(1)}=\delta \bar{R}_{\mathrm{b}}^{(3)}-\left(\delta \bar{R}_{\mathrm{b}}^{(3)}-\delta \bar{R}_{\mathrm{b}}^{(2)}\right) \frac{\mathrm{t}_{3}-\mathrm{t}_{1}}{\mathrm{t}_{3}-\mathrm{t}_{2}}, \\
& \delta \bar{R}_{\mathrm{b}}^{(n)}=\delta \bar{R}_{\mathrm{b}}^{(n-2)}-\left(\delta \bar{R}_{\mathrm{b}}^{(n-1)}-\delta \bar{R}_{\mathrm{b}}^{(n-2)}\right) \frac{t_{n}-t_{n-2}}{t_{n-1}-t_{n-2}} .
\end{aligned}
$$


Depending on the level of noise this rounding procedure should be repeated until a smooth $\delta R_{\mathrm{b}}(t)$ curve is obtained. Differently than procedure used in Ref. [1] the described method of reduction of noise accounts for the level of noise.

\section{Results of calculations}

\subsection{Example of deconvolution procedure}

We performed the deconvolution of the phonon signal obtained in experiments performed by Danilchenko and collaborators [10] (Fig. 2). In these experiments long wavelength acoustic phonons were generated in GaAs specimen by $1 \times 1 \mathrm{~mm}^{2}$ Au film heated by electric pulses each of $75 \mathrm{~ns}$ duration. Beams of phonons propagated in the (001) direction. The thickness of substrate was $3.4 \mathrm{~mm}$, this allowed to resolve longitudinal acoustic (LA) and transversal acoustic (TA) phonon modes. First, the phonoconductivity of two-dimensional electron gas (2DEG) was measured. The 2DEG structure of area $2 \times 0.2 \mathrm{~mm}^{2}$ was formed by 7 layers of the delta-doped GaAs. The carrier concentration in each layer was $5 \times 10^{11} \mathrm{~cm}^{-2}[10]$. For this method of registration of phonon pulses the response time is smaller than $5 \mathrm{~ns}$. Then, by the direct evaporation of indium film on the 2DEG mesostructure, the bolometer of sensitive area $2 \times 0.2 \mathrm{~mm}^{2}$ was fabricated. Phonons were detected by this superconducting detector. Thus, we had an unique opportunity to compare the deconvoluted bolometer signal with signals obtained in experiments of quite different types. The 2DEG structure and the bolometer have

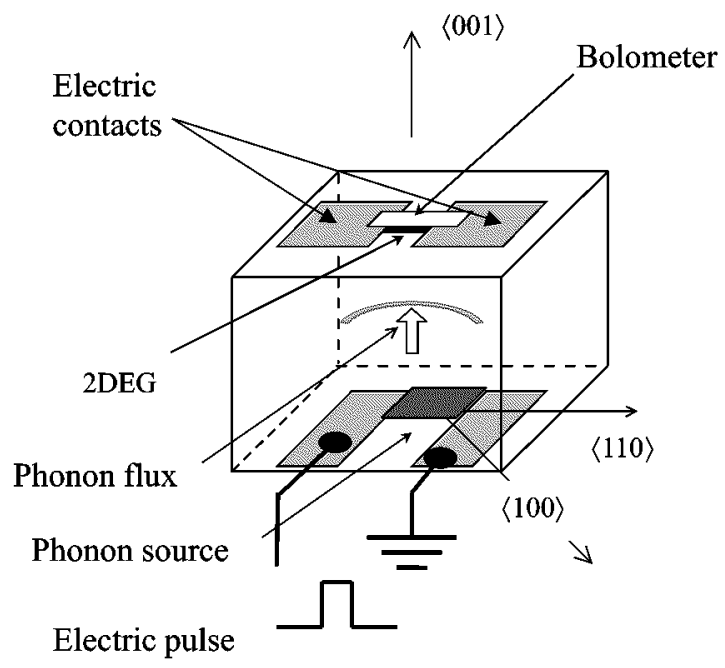

Fig. 2. In our experiments the response of $2 \mathrm{DEG}$ and of superconducting bolometer is measured. 


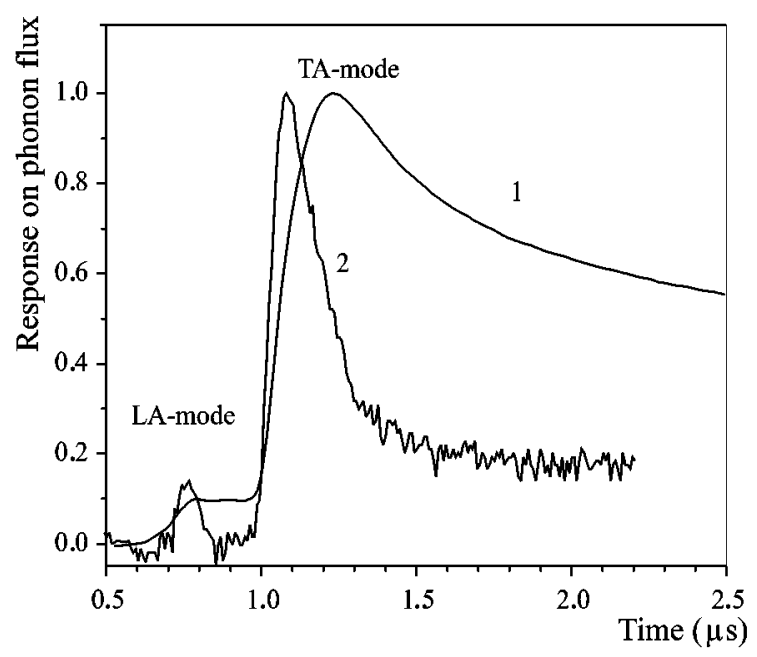

Fig. 3. Response of superconducting bolometer (1) and of 2DEG (2) to the same phonon flux. Phonons propagate in the (001) direction of GaAs. Maxima of both curves are normalized to unity.

common contact area. However, the large resistance of $2 \mathrm{DEG}(\approx 500 \Omega)$ prevents the short circuit of the bolometer (its resistance is $\approx 20 \Omega$ ). We should underline that in these two different kinds of experiments the phonons were generated by the same source. Additionally the geometrical factors in these experiments were identical (cf. Fig. 2). The curve with several sharp peaks seen in Fig. 3 represents results of two above-mentioned experiments. The curve with narrow maxima represents time dependent phonoconductivity, while the broader ones correspond to the bolometer response $R_{\mathrm{b}}(t)$. Due to phonon focusing one sees the weak signal related to longitudinal phonons and strong signals produced by transverse phonons. The width of signal of the bolometer is related to the heat inertia. In comparison with the bolometer signal the large maximum for the phonoconductivity is narrow. As a result of the frequency cut mechanisms (characteristic of interaction of 2D carriers with bulk acoustic phonons) the phonoconductivity is built only by ballistic phonons [13]. The inertia of the response of 2DEG is related mainly to the time constant of the electric circuit, being in our experiments of the order of 5 ns. In contrast slow and scattered phonons give rise to the tail of the bolometer signal.

In Fig. 4 we present the comparison of results of the procedure of deconvolution, described in previous section, with the results of Monte Carlo simulations performed on the specimen, source, and detectors used in real experiments. We assumed that the source of phonons is Planckian with temperature $10 \mathrm{~K}$, and we accounted for the finite duration of current pulses $75 \mathrm{~ns}$, as well as for the focusing effects, elastic scattering on isotopes and anharmonic down-conversion processes [12]. 


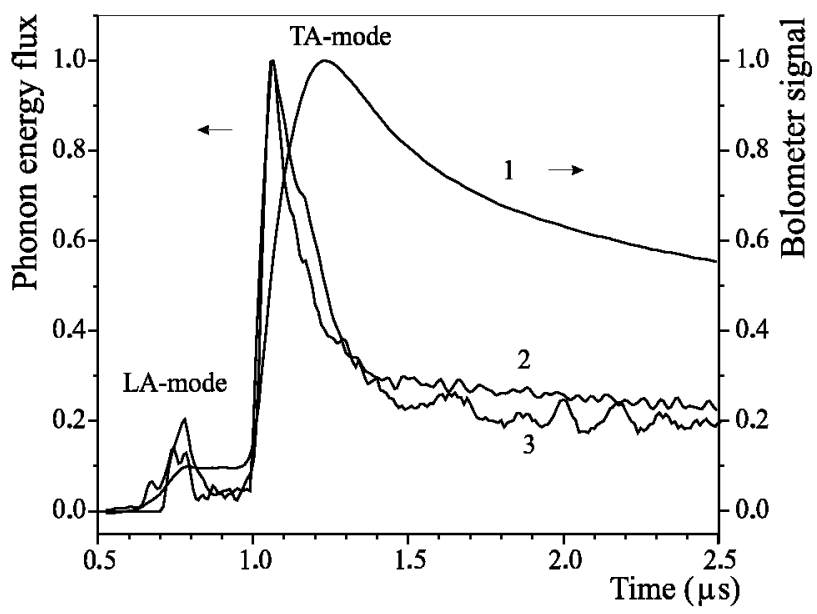

Fig. 4. Comparison of the experimentally obtained bolometer signal (1) with the deconvoluted phonon flux (2), and with the results of Monte Carlo simulations (3). Maxima of all curves are normalized to unity.

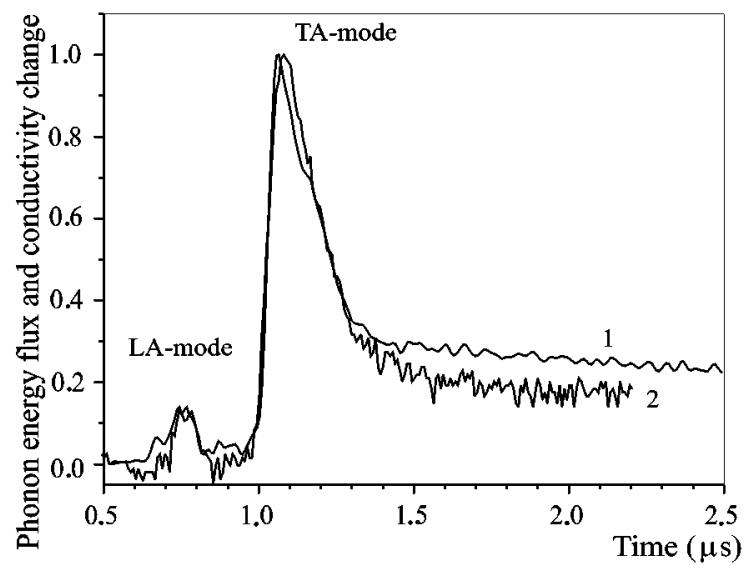

Fig. 5. Comparison of the deconvoluted phonon flux (1) with the experimentally obtained phonoconductivity (2). Maxima of both curves are normalized to unity.

In Fig. 5 we compare the time response of 2DEG structure with deconvoluted phonon flux. For the deconvoluted phonon flux the best fit was obtained for $A=$ $0.25 \mu \mathrm{s}$ (Eq. (7)). The inspection of Figs. 4, 5 shows that the leading fronts and the arrival times of maxima of curves obtained by these three independent methods agree quite well. Let us notice, however, that our method does not reproduce the decaying part of phonon flux equally well. In particular the peak is too broad. We expect that accounting for nonlinearity related to heat exchange should improve results of deconvolution. 


\subsection{Example of solution of the inverse problem}

We also solved the inverse problem. Namely, using our Monte Carlo results obtained for phonon energy flux with the help of Eq. (11) we calculated the corresponding signal of bolometer. The maximum of this calculated signal is normalized to unity and depends only on $A$. Results are shown in Fig. 6 , a satisfactory agreement was obtained for $\Lambda=0.25 \mu \mathrm{s}$.

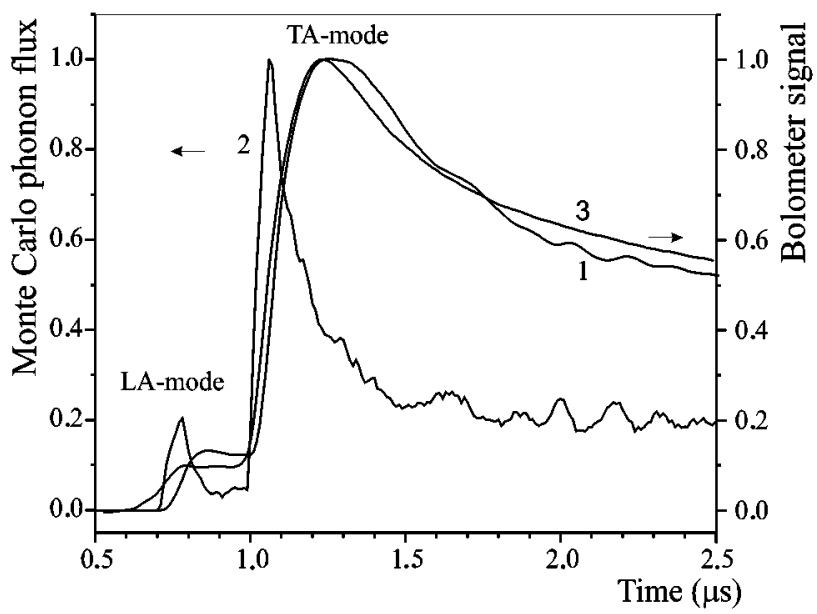

Fig. 6. Comparison of results of the convolution procedure (1) applied to the Monte Carlo results (2) with the bolometer signal measured in our experiments (3). Maxima of all curves are normalized to unity.

\section{Conclusions}

We developed the effective procedure of deconvolution of the phonon flux. This procedure gives the phonon fluxes if signals of metal films bolometers (superconducting as well as in normal state) are measured. The suitable algorithm is based on the linear differential equation for the resistivity $R_{\mathrm{b}}(t)$ of the bolometer. The inverse problem of obtaining the bolometer resistance using results of numerical calculations, described in Sec. 5, or Monte Carlo computer experiments [12] is also solved.

The obtained results are consistent: the times characterizing phonoconductivity signal and phonon fluxes - obtained as a result of Monte Carlo experiments and deconvoluted, are of the order of several nanoseconds. The signals of bolometer obtained in real experiments and calculated by procedure of deconvolution, are characterized by the same time scales - their fronts by nanoseconds, their tails by $10^{-1} \mu$ s.

The basic constants $C, G$ characterizing the particular bolometers are not accessible directly and are quite difficult to calculate. The analysis of solutions of 
the basic Eq. (6) performed in Sec. 4 suggests experiments in which these constants can be deduced. In accordance with Fuson's results [3] we noticed that the effective resistance relaxation rate depends on the ratio of the bias current $I_{\mathrm{b}}$ and the characteristic current $I_{\mathrm{m}}$. This characteristic current depends on the thermal characteristics of the particular bolometer. Our deconvolution method allows to find the crucial parameters $\Lambda$ and $I_{\mathrm{m}}$.

Fits of these parameters can be improved if one compares the deconvolution results with the Monte Carlo results or other independent measurements. The effectiveness of our deconvolution procedure is demonstrated. In a consistent way we compared results of deconvolution with results of Monte Carlo simulations (performed for models close to real systems) and measurements of phonoconductivity. Measurements of phonoconductivity allows to study the response of $2 \mathrm{D}$ electron gas - a subsystem which is not affected by the constants $\alpha, C, G$ characterizing the bolometer.

\section{Acknowledgments}

This work was partially supported by the State Committee for Scientific Research (Poland) under grant No. 7 T08A 00821 and by CRDF. B.D. also acknowledges the State Committee for Scientific Research for financing his visits to Poland. One of us (T.P.) is grateful to Professor J.K. Wigmore, who several years ago brought our attention to the discussed problem, for his hospitality making visit to University of Lancaster pleasant and fruitful. We also would like to acknowledge Tony Kent and Madeleine Msall for discussions and Miles Blencowe for the help in finding Fuson's paper.

\section{References}

[1] S.C. Edwards, Hamid bin Rani, J.K. Wigmore, J. Phys. E, Sci. Instrum. 22, 528 (1989).

[2] B.A. Danilchenko, W.M. Gańcza, Cz. Jasiukiewicz, T. Paszkiewicz, Phys. Rev. B 60, 6113 (1999).

[3] N. Fuson, J. Appl. Phys. 20, 59 (1949).

[4] R.J. von Gutfeld, in: Physical Acoustics Principles and Methods, Vol. 5, Ed. W.P. Mason, Academic Press, New York 1968.

[5] R.A. Sherlock, J. Phys. E, Sci. Instrum. 17, 386 (1984).

[6] G.A. Northrop, J.P. Wolfe, in: Nonequilibrium Phonons, Ed. W.A. Bron, Plenum Press, New York 1985, p. 165.

[7] J.P. Wolfe, Imaging Phonons, Acoustic Wave Propagation in Solids, Cambridge University Press, Cambridge 1998.

[8] K.D. Irvin, Appl. Phys. Lett. 66, 1998 (1995).

[9] K.D. Irvin, G.C. Hilton, D.A. Wollman, J.M. Martinis, Appl. Phys. Lett. 69, 1945 (1996) 
[10] B. Danilchenko, A. Klimashov, O. Sarbey, M. Asche, R. Hey, in: Proc. 23rd Int. Conf. on Physics of Semiconductors, Eds. M. Scheffler, R. Zimmermann, Vol. 3, World Sci., Singapore 1996, p. 2339.

[11] B.A. Danilchenko, M.I. Slutskii, Sov. Phys.-Solid State 30, 21 (1988).

[12] W.M. Gańcza, I.A. Obukhov, T. Paszkiewicz, B.A. Danilchenko, Comput. Meth. Sci. Technol. 7, 7 (2001).

[13] D.V. Poplavsky, B.A. Danilchenko, H. Kostial, Phys. Rev. B 61, 10941 (2000). 